\title{
Incremental Techniques for Large-Scale Dynamic Query Processing
}

\author{
Iman Elghandour \\ Université Libre de Bruxelles \\ ielghand@ulb.ac.be \\ Dan Olteanu \\ University of Oxford \\ dan.olteanu@cs.ox.ac.uk
}

\author{
Ahmet Kara \\ University of Oxford \\ ahmet.kara@cs.ox.ac.uk \\ Stijn Vansummeren \\ Université Libre de Bruxelles \\ svsummer@ulb.ac.be
}

\begin{abstract}
Many applications from various disciplines are now required to analyze fast evolving big data in real time. Various approaches for incremental processing of queries have been proposed over the years. Traditional approaches rely on updating the results of a query when updates are streamed rather than re-computing these queries, and therefore, higher execution performance is expected. However, they do not perform well for large databases that are updated at high frequencies. Therefore, new algorithms and approaches have been proposed in the literature to address these challenges by, for instance, reducing the complexity of processing updates. Moreover, many of these algorithms are now leveraging distributed streaming platforms such as Spark Streaming and Flink. In this tutorial, we briefly discuss legacy approaches for incremental query processing, and then give an overview of the new challenges introduced due to processing big data streams. We then discuss in detail the recently proposed algorithms that address some of these challenges. We emphasize the characteristics and algorithmic analysis of various proposed approaches and conclude by discussing future research directions.
\end{abstract}

\section{CCS CONCEPTS}

- Theory of computation $\rightarrow$ Lower bounds and information complexity; Database query processing and optimization (theory); Distributed computing models;

\section{KEYWORDS}

Dynamic query processing; Incremental View Maintenance; Big Data; Data Streams;

\section{ACM Reference Format:}

Iman Elghandour, Ahmet Kara, Dan Olteanu, and Stijn Vansummeren. 2018. Incremental Techniques for Large-Scale Dynamic Query Processing. In The 27th ACM International Conference on Information and Knowledge Management (CIKM '18), October 22-26, 2018, Torino, Italy. ACM, New York, NY, USA, 2 pages. https://doi.org/10.1145/3269206.3274271

Permission to make digital or hard copies of part or all of this work for personal or classroom use is granted without fee provided that copies are not made or distributed for profit or commercial advantage and that copies bear this notice and the full citation on the first page. Copyrights for third-party components of this work must be honored.

For all other uses, contact the owner/author(s)

CIKM '18, October 22-26, 2018, Torino, Italy

(C) 2018 Copyright held by the owner/author(s).

ACM ISBN 978-1-4503-6014-2/18/10.

https://doi.org/10.1145/3269206.3274271

\section{INTRODUCTION}

In a broad range of domains, such as Real Time Business Intelligence and Complex Event Processing, contemporary applications require the timely dynamic processing of complex analytical queries on continuously arriving data. Here, dynamic processing refers to updating the query result, preferably in real-time, when the underlying data is updated. Implementing such applications remains a difficult task, and involves resolving two orthogonal challenges:

- Designing a suitable dynamic query processing algorithm that determines how the application's query results are to be updated upon data changes, taking into account that previous results are already available and re-computation should be avoided to ensure timeliness.

- Designing an implementation and deployment of the selected dynamic query processing algorithm that accounts for desiderata such as high throughput, low latency, and the ability to process large data sets. Current approaches mostly rely on distributed computing frameworks such as MapReduce, Flink, Spark, or Storm, to achieve this.

Fortunately, in recent years, there has been a flurry of research on both challenges that provide novel insights in how to resolve them. We briefly survey these next.

Algorithmical insights. Avoiding the re-computation whenever an update is received has long been approached using Incremental View Maintenance (IVM) techniques [6, 9]. IVM materializes the output of a query and then maintains that output under updates. Unfortunately, traditional IVM is not efficient for large databases that are updated at a high frequency. Therefore, new approaches have recently been proposed, whose objective is to reduce the complexity of processing updates and/or to reduce the required memory footprint.

Specifically, research in dynamic query processing has recently received a big boost with: (1) the introduction of Higher-Order IVM (HIVM) [13, 14, 19]; (2) the identification of lower bounds and worstcase optimal algorithms for processing updates [3, 4, 12]; (3) the practical formulations of worst-case-optimal IVM that implement and extend these algorithms $[10,11,20]$; and (4) the introduction of the notion of differential dataflow for computations that require recursive or iterative processing [16, 17]. These approaches often rely on materializing a succinct representation of a query's output to maintain it more efficiently, and therefore present a fundamental breakthrough with traditional IVM techniques. 
Big Data Frameworks support for dynamic query processing. Big data frameworks such as MapReduce [7] and Spark [24] are inherently batch-oriented. Early approaches for implementing dynamic query processing in these frameworks has focused on incremental processing of MapReduce tasks [5, 21, 23]. These are, however, based on traditional IVM techniques and suffer from high latency of MapReduce and its open source implementation Hadoop More recent versions of distributed compute frameworks such as Apache Spark [25], Apache Flink [1], and Twitter Storm [22] / Heron [15] allow stream-based computations instead of batch-based computations. Out of the box, these frameworks mostly provide primitives for avoiding re-computation over sliding windows, based on traditional IVM. In addition, they present low-level programming primitives by which developers can express their own dynamic query processing algorithms. More recently, there are proposals to automatically incrementalize queries on distributed big data frameworks. Examples of these approaches include the distributed implementation of HIVM [18] and differential dataflow [17], as well as Spark Sructured Streaming [2].

\section{TUTORIAL STRUCTURE}

The tutorial runs for 3 hours and is divided into the following four parts:

\section{Part I: Introduction, desiderata, and traditional IVM}

We start the tutorial by giving an introduction to dynamic query processing and show examples that motivate the need for efficient incremental query processing. We give a high-level historical overview of traditional approaches (known as First Order IVM) that have been employed by conventional database systems to maintain query outputs. We present the strong and weak points of traditional approaches and then discuss new challenges introduced by streaming large data at high frequencies.

\section{Part II: Recent Algorithmic Advances in Dynamic Query Pro- cessing}

In the second part of the tutorial, we survey new efficient approaches and algorithms for dynamic query processing. We discuss the following research works: (1) Higher-Order IVM [13, 14, 19]; (2) Complexity lower bounds for dynamic query processing [3, 4, 12]; (3) Dynamic Yannakakis [10, 11]; (4) Factorized IVM [20]; (5) Spacetime tradeoffs [12]; and (6) Beyond conjunctive queries: relations over application-dependent rings [8, 13, 20].

Part III: Dynamic Query Processing in Big Data Frameworks Incremental processing of queries has been studied for queries executed by MapReduce [5, 21, 23] and by other distributed streaming platforms such as Spark Streaming [2, 25], Flink [1], and Storm [22]/Heron [15]. However, these systems rely on their users to specify how the queries are maintained or employ traditional incremental view maintenance approaches. Additionally, new parallel approaches that are executed in distributed environments [18] or that extend incremental processing [17] are introduced. We discuss all the mentioned approaches and platforms while highlighting the contributions that each one of them has made.

\section{Part IV: Outlook}

Finally we conclude by summarizing the existing research solutions and highlighting the open problems that are yet to be studied.

\section{ACKNOWLEDGMENT}

The authors are graciously supported by the Wiener-Anspach foundation. The work has received funding from the European Union's Horizon 2020 research and innovation programme under grant agreement 682588 .

\section{REFERENCES}

[1] Alexander Alexandrov et al. 2014. The Stratosphere Platform for Big Data Analytics. The VLDB fournal 23, 6 (Dec. 2014), 939-964.

[2] Michael Armbrust et al. 2018. Structured Streaming: A Declarative API for Real-Time Applications in Apache Spark. In Proc. ACM SIGMOD Int. Conf. on Management of Data. 601-613.

[3] Christoph Berkholz, Jens Keppeler, and Nicole Schweikardt. 2017. Answering Conjunctive Queries under Updates. In Proc. ACM SIGACT-SIGMOD-SIGART Symp. on Principles of Database Systems (PODS). 303-318.

[4] Christoph Berkholz, Jens Keppeler, and Nicole Schweikardt. 2018. Answering UCQs under Updates and in the Presence of Integrity Constraints. In Proc. ACM Int. Conf. on Database Theory (ICDT). 8:1-8:19.

[5] Pramod Bhatotia, Alexander Wieder, Rodrigo Rodrigues, Umut A Acar, and Rafael Pasquin. 2011. Incoop: MapReduce for Incremental Computations. In Proc. ACM Symposium on Cloud Computing. 7:1-7:14.

[6] Rada Chirkova and Jun Yang. 2012. Materialized Views. Found. \& Trends in DB 4, 4 (2012), 295-405.

[7] Jeffrey Dean and Sanjay Ghemawat. 2004. MapReduce: Simplified Data Processing on Large Clusters. In Proc. USENIX Conf. on Operating Systems Design and Implementation (OSDI). 137-150.

[8] Todd J. Green, Grigoris Karvounarakis, and Val Tannen. 2007. Provenance Semirings. In Proc. ACM SIGACT-SIGMOD-SIGART Symp. on Principles of Database Systems (PODS). 31-40.

[9] Ashish Gupta and Iderpal Singh Mumick (Eds.). 1999. Materialized Views: Techniques, Implementations, and Applications. MIT Press, Cambridge, MA, USA.

[10] Muhammad Idris, Martín Ugarte, and Stijn Vansummeren. 2017. The Dynamic Yannakakis Algorithm: Compact and Efficient Query Processing Under Updates. In Proc. ACM SIGMOD Int. Conf. on Management of Data. 1259-1274.

[11] Muhammad Idris, Martín Ugarte, Stijn Vansummeren, Hannes Voigt, and Wolfgang Lehner. 2018. Conjunctive Queries with Inequalities Under Updates. Proc. VLDB Endow. (PVLDB) 11, 7 (2018), 733-745.

[12] Ahmet Kara, Hung Q. Ngo, Milos Nikolic, Dan Olteanu, and Haozhe Zhang. 2019. Counting Triangles under Updates in Worst-Case Optimal Time. In ICDT (to appear).

[13] Christoph Koch. 2010. Incremental query evaluation in a ring of databases. In Proc. ACM SIGACT-SIGMOD-SIGART Symp. on Principles of Database Systems (PODS). 87-98.

[14] Christoph Koch, Yanif Ahmad, Oliver Kennedy, Milos Nikolic, Andres Nötzli, Daniel Lupei, and Amir Shaikhha. 2014. DBToaster: higher-order delta processing for dynamic, frequently fresh views. The VLDB fournal 23, 2 (2014), 253-278.

[15] Sanjeev Kulkarni et al. 2015. Twitter Heron: Stream Processing at Scale. In Proc. ACM SIGMOD Int. Conf. on Management of Data. 239-250.

[16] Frank McSherry, Derek Gordon Murray, Rebecca Isaacs, and Michael Isard. 2013. Differential Dataflow. In Proc. Conf. on Innovative Data Systems Research (CIDR).

[17] Derek G. Murray, Frank McSherry, Michael Isard, Rebecca Isaacs, Paul Barham, and Martin Abadi. 2016. Incremental, Iterative Data Processing with Timely Dataflow. Commun. ACM 59, 10 (Sept. 2016), 75-83.

[18] Milos Nikolic, Mohammad Dashti, and Christoph Koch. 2016. How to Win a Hot Dog Eating Contest: Distributed Incremental View Maintenance with Batch Updates. In Proc. ACM SIGMOD Int. Conf. on Management of Data. 511-526.

[19] Milos Nikolic, Mohammed Elseidy, and Christoph Koch. 2014. LINVIEW: incremental view maintenance for complex analytical queries. In Proc. ACM SIGMOD Int. Conf. on Management of Data. 253-264.

[20] Milos Nikolic and Dan Olteanu. 2018. Incremental View Maintenance with Triple Lock Factorization Benefits. In Proc. ACM SIGMOD Int. Conf. on Management of Data. 365-380.

[21] Christopher Olston et al. 2011. Nova: continuous pig/hadoop workflows. In Proc. ACM SIGMOD Int. Conf. on Management of Data. 1081-1090.

[22] Ankit Toshniwal et al. 2014. Storm@Twitter. In Proc. ACM SIGMOD Int. Conf. on Management of Data. 147-156.

[23] Cairong Yan, Xin Yang, Ze Yu, Min Li, and Xiaolin Li. 2012. IncMR: Incremental Data Processing Based on MapReduce. In Proc. IEEE Int. Conf. on Cloud Computing. 534-541.

[24] Matei Zaharia et al. 2012. Resilient Distributed Datasets: A Fault-tolerant Abstraction for In-memory Cluster Computing. In USENIX Conf. on Networked Systems Design and Implementation (NSDI). 15-28.

[25] Matei Zaharia, Tathagata Das, Haoyuan Li, Timothy Hunter, Scott Shenker, and Ion Stoica. 2013. Discretized Streams: Fault-tolerant Streaming Computation at Scale. In Proc. ACM Symp. on Operating Systems Principles (SOSP). 423-438. 